\title{
Diagnostic accuracy of Leucine-rich a-2 glycoprotein (LRG) as a urinary biomarker in pediatric appendicitis: a prospective observational pilot study from Eastern India
}

Santosh Kumar Mahalik ${ }^{1 *}$ D, Debapriya Bandyopadhyay ${ }^{2}$, Bikasha Bihary Tripathy ${ }^{1}$, Akash Bihari Pati ${ }^{1}$ and Manoj Kumar Mohanty ${ }^{1}$

\begin{abstract}
Background: Recently, several serum and urinary biomarkers have been investigated for diagnosis of acute appendicitis. Urinary biomarkers seem to be advantageous in children as it is non-invasive, painless, and easy to perform. Leucine-rich a-2-glycoprotein $(L R G)$ is regarded as a reliable urinary biomarker for the diagnosis of pediatric appendicitis. A prospective observational pilot study was conducted in children presenting with abdominal pain. Assessment of pediatric appendicitis score (PAS), routine blood tests, and measurements of urinary LRG was done. The present study aimed to evaluate the diagnostic accuracy of urine LRG in appendicitis in Indian children and to assess the concentration of urine LRG at which it will guide the management.

Results: LRG had a receiver operating characteristic (ROC) area under the curve (AUC) of 0.586 (95\% Cl 0.4070.766). There was no specific cut-off identified using Youden's index. ROC analysis of the PAS score resulted in an AUC of 0.821 (95\% Cl 0.691-0.952). Using Youden's index, the cut-off for PAS scoring was identified as 6.5 with the sensitivity of $80 \%$, the specificity of $76.2 \%$, positive predictive value $76.19 \%$, negative predictive value as $80 \%$, and diagnostic accuracy of $78 \%$.
\end{abstract}

Conclusions: LRG could not show better diagnostic performance compared to routine blood tests and PAS scores. There was no specific cut-off value at which it will differentiate mesenteric adenitis and acute appendicitis and guide their management. With 78\% diagnostic accuracy, PAS score $(>6)$ is still a better tool for the diagnosis of acute appendicitis.

Trial registration: Clinical Trials Registry Government of India, CTRI/2018/01/011182, Registered on: 08 Jan 2018. Keywords: Appendicitis, Mesenteric lymphadenitis, Biomarkers, LRG, PAS

\footnotetext{
* Correspondence: dr.santosh25@gmail.com

${ }^{1}$ Department of Pediatric Surgery, All India Institute of Medical Sciences (AllM

S) Bhubaneswar, Sijua, Patrapada, Bhubaneswar, Odisha 751020, India

Full list of author information is available at the end of the article
}

\section{Springer Open}

(- The Author(s). 2021 Open Access This article is licensed under a Creative Commons Attribution 4.0 International License, which permits use, sharing, adaptation, distribution and reproduction in any medium or format, as long as you give appropriate credit to the original author(s) and the source, provide a link to the Creative Commons licence, and indicate if changes were made. The images or other third party material in this article are included in the article's Creative Commons licence, unless indicated otherwise in a credit line to the material. If material is not included in the article's Creative Commons licence and your intended use is not permitted by statutory regulation or exceeds the permitted use, you will need to obtain permission directly from the copyright holder. To view a copy of this licence, visit http://creativecommons.org/licenses/by/4.0/. 


\section{Background}

Accurate clinical diagnosis of pediatric appendicitis remains a challenge and a perfect test is yet to be found. Mesenteric lymphadenitis is an important differential diagnosis in children as it clinically mimics appendicitis. Mesenteric lymphadenitis has been reported as the most common finding during negative surgical explorations for suspected appendicitis [1]. PAS is a specific clinical scoring system for children and incorporates 8 variables. Score $\geq 6$ is indicative of a high probability of acute appendicitis [2]. However, even with PAS score $\geq 8$, there is a $5 \%$ rate of negative appendectomy [3]. Advanced imaging studies such as ultrasonography (US) and computed tomography (CT) are therefore often used to confirm or rule out appendicitis. Ultrasonography is highly operator dependent and may not be available in odd hours, particularly at night at many hospitals. Similarly, CT imaging may not be available at many hospitals. Furthermore, CT imaging has issues of ionizing radiation which in children increases the lifetime mortality risk from cancer [4]. Therefore, CT scans in children should be avoided whenever possible.

Recently, several serum and urinary biomarkers have been investigated for diagnosis of acute appendicitis. These are biomarkers of inflammation and reported to be helpful in diagnosis. Urine analysis is non-invasive, painless, and easy to perform; hence, urinary biomarkers seem to be advantageous in children. Out of various urinary biomarkers, Leucine-rich $\alpha$-2-glycoprotein (LRG) has shown the best results in three previously done studies in children [5-7]. According to Salo et al., urine LRG when adjusted for dehydration is a promising biomarker for differentiating between patients with or without appendicitis and for evaluating the severity of the disease [7]. LRG used in conjunction with PAS provides high predictive values [7].

\section{Objectives}

The actual prevalence and incidence of appendicitis in Indian children are not known, and there was no study on urinary biomarkers for appendicitis from India. Therefore, the study aimed to evaluate the diagnostic accuracy of urine LRG in pediatric appendicitis and whether it can differentiate between appendicitis and mesenteric lymphadenitis. We also aimed to assess the concentration of urine LRG at which it will identify and differentiate patients with appendicitis who will need conservative management and surgery.

\section{Methods}

A prospective observational pilot study was designed to validate the diagnostic accuracy of urine LRG in an independent cohort of pediatric and adolescent patients (age 3-16 years) presenting to the outpatient department
(OPD) and emergency department (ED) with abdominal pain mimicking acute appendicitis at our tertiary teaching hospital. Data were collected from December 2017 to April 2019. As the incidence of appendicitis and mesenteric lymphadenitis is not known in India, a formal sample size calculation was not done.

All consecutive patients presenting with abdominal pain - central, right lower quadrant, shifting of pain or generalized pain, and associated symptoms such as nausea, vomiting, and fever with a duration of symptoms less than or equal to $72 \mathrm{~h}$ (from onset) were included in the study. Written informed consent was obtained from the parent or guardian. Children having urinary tract infection, pyelonephritis, pneumonia, treatment with immune-modulating therapy, prior abdominal surgery, and chronic illness (e.g., diabetes, chronic pancreatitis, inflammatory bowel disease, cystic fibrosis, sickle cell anemia) were excluded from the study. The study was approved by the local institutional ethics committee (T/ IM-F/17-18/36). The study was also enrolled in the Clinical Trials Registry of Government of India with registration number CTRI/2018/01/01118.

\section{Study protocol}

All patients presenting to the OPD and ED with abdominal pain mimicking acute appendicitis were enrolled. History and physical examination data were recorded in a structured case report form specifically designed for this study. The treating physicians complete the form before sending them for any radiological study (US or CT). Preoperative diagnosis of acute appendicitis was based on history, clinical examination, blood test (Complete blood count [CBC], especially increased Leukocyte count, absolute neutrophil count [ANC]), and by ultrasound examination. Acute appendicitis is considered when the appendix diameter is $>6 \mathrm{~mm}$, noncompressible, increase vascularity, presence of target sign, and appendicolith [8]. Mesenteric lymphadenitis is considered when there is the presence of 3 or more hypervascular mesenteric lymph node of size $>5 \mathrm{~mm}$ in short axis and absence of other parameters of appendicitis $[1,8]$. A pediatric appendicitis score (PAS) was recorded for each patient. The patients' attendants have explained the procedure of the collection of urinary samples. Urine samples were collected in a sterile urine container, then aliquoted into two sterile labeled microcentrifuge tubes before storing at $-20{ }^{\circ} \mathrm{C}$ within $1 \mathrm{~h}$ of collection. The urine samples were analyzed after inclusion in the study. The decision to operate or manage conservatively was always based on the clinical judgment of the surgeon and the radiological findings. In general, all uncomplicated appendicitis cases were managed conservatively and those who did not improve, and those 
with features of complicated appendicitis were managed with surgery.

Patients with mesenteric lymphadenopathy and other patients with a diagnosis of uncomplicated acute appendicitis (by clinico-radiologic examinations) but managed conservatively were followed clinically until discharge and after 1 month of discharge.

\section{Urine analysis for LRG}

Before analyses, urine samples were thawed to room temperature and centrifuged ( $3500 \mathrm{RPM}$ for $10 \mathrm{~min}$ ). One of the tubes was used for estimation of urinary creatinine on Beckman Coulter AU5800 fully automated analyzer, by kinetic modification of Jaffe's procedure, using reagents from Beckman Coulter. The second tube was used to estimate urinary LRG in batches by sandwich ELISA kits from ASSAYPRO, USA, using automated BIOTEK washer and reader by the investigator from the Department of Biochemistry who was blinded to patient information.

\section{Outcome}

Clinical outcome was defined as mesenteric lymphadenitis or acute appendicitis and appendicitis treated by conservative management or surgery. The laboratory outcome was defined as the concentration of urine LRG at which it will differentiate between mesenteric lymphadenitis and acute appendicitis and concentration of urine LRG (range) in different types of acute appendicitis (phlegmonous, gangrenous, perforated).

\section{Statistical analysis}

Patient data were recorded in an excel database. WBC and absolute neutrophil count (ANC) were converted to dichotomous variable, i.e., yes or no, for leucocytosis and neutrophilia. Urine LRG concentrations were divided by urine creatinine concentration, to adjust for different grades of dehydration among patients. Assessment of association between urine LRG and the presence or absence of appendicitis was done using Fisher's exact test for the dichotomous variables and by two-tailed MannWhitney $U$ test for continuous variables, as urine LRG levels are expected to be asymmetrically distributed. Receiver operator characteristic (ROC) curves with an AUC and 95\% confidence interval (CI) was performed to explore the performance of urine LRG, PAS to predict appendicitis (complicated and uncomplicated). From the ROC curve data, the best cut-off values was selected by choosing a value that maximized both sensitivity and specificity (Youden's index). From this cut-off value, the positive predictive value (PPV) and negative predictive value $(\mathrm{NPV})$ were calculated. Statistical significance was set at $p<0.05$.

\section{Results}

In total, 61 patients were enrolled in the study. Out of which, 20 patients were excluded due to incomplete data entry/investigations or other diagnoses such as UTI, colitis, and stones in the urinary tract. Out of the 41 cases, 31 cases were male and 10 cases were female. Twenty patients were diagnosed as acute appendicitis and 21 were diagnosed as mesenteric lymphadenitis. Thirteen patients of acute appendicitis were managed conservatively and all were improved. Seven patients underwent surgery. Five had complicated appendicitis such as gangrenous appendicitis, appendicular perforation, and abscess. Two patients had uncomplicated appendicitis with an inflamed appendix.

Both appendicitis and mesenteric lymphadenitis groups were comparable and there was no significant difference found regarding the age of the children, TLC ANC, urinary LRG, and dehydration-corrected urinary LRG (Table 1). Urinary LRG was found to be significantly elevated in 4 cases of mesenteric adenitis which were managed conservatively and improved without any recurrence. ROC curve analysis of LRG resulted in an AUC of 0.586 (95\% CI 0.407-0.766) (Fig. 1). There was no specific cut-off identified using Youden's index. Hence, the predictive values of LRG were not calculated. When dehydration-corrected LRG was compared between the appendicitis patients who underwent surgery and the others who were managed conservatively, the $p$ value was 1.0, i.e, non-significant. ROC analysis of TLC and ANC resulted in an AUC of 0.401 and 0.297 respectively which is inferior to the AUC of LRG, i.e., 0.586 . However, all had poor diagnostic performances. ROC curve analysis of the PAS score resulted in an AUC of 0.821 (95\% CI 0.691-0.952) (Fig. 2). Using Youden's index, the cut-off for PAS scoring was identified as 6.5 with the sensitivity of $80 \%$, the specificity of $76.2 \%$, positive predictive value $76.19 \%$, negative predictive value as $80 \%$, and diagnostic accuracy of $78 \%$. The diagnostic accuracy of combined LRG and PAS were only $26.8 \%$.

\section{Discussion}

Leucine-rich $\alpha$-2-glycoprotein (LRG) is a glycoprotein belonging to the leucine-rich- repeat family of proteins that are involved in signal transduction, cell adhesion, and protein-protein interaction. It is expressed by neutrophils undergoing differentiation, by the liver, and in high endothelial venules of mesentery such as mesoappendix and thought to play a role in the activation and/ or chemotaxis of neutrophils as they enter the area of inflammation, which is the principal pathway of appendicitis [7].

Kentis et al. [5] found that LRG is enriched in the diseased appendix and elevated in the urine of patients with 
Table 1 Demography, blood, and urine test result of included children

\begin{tabular}{llll}
\hline & Mesenteric lymphadenitis & Acute appendicitis & $\boldsymbol{P}$-value \\
\hline Total & 21 & 20 & 14 \\
Gender, male & 18 & $10.2 \pm 3.63$ & 0.584 \\
Age (year) & $9.59 \pm 3.37$ & $12847 \pm 5509$ & 0.127 \\
TLC & $10543 \pm 3650$ & $9493 \pm 6741$ & 0.258 \\
ANC & $7180 \pm 3842$ & $8.57 \pm 8.024$ & 0.456 \\
Urinary LRG & $6.68 \pm 7.848$ & $0.2818 \pm 0.395$ & 0.593 \\
Corrected LRG & $0.2215 \pm 0.309$ & &
\end{tabular}

Values presented as mean \pm standard deviation (SD), $P$-value $<0.05$ (significant)

$T L C$ total leucocyte count, $A N C$ absolute neutrophil count, $L R G$ leucine-rich a-2-glycoprotein

appendicitis, including patients without radiographic evidence of appendicitis. They also found out that LRG correlates with the severity of appendicitis [6]. However, the assay method was reported to play an important role in the outcome. When measured by ELISA, an immunoassay interference effect was described and AUC of 0.80 was reached. When it was evaluated by mass spectrometry, the AUC reached 0.98-0.99 which is highly effective [5]. Kharbanda et al. [6] evaluated urinary LRG via commercially available ELISA kit in his study on 137 children and found no difference between LRG in patients with phlegmonous appendicitis compared to patients without appendicitis. However, there was a significant difference between perforated and non-perforated appendicitis. The ROC AUC for LRG was 0.63 and showed $100 \%$ sensitivity, $23 \%$ specificity, and $100 \%$ NPV [6].

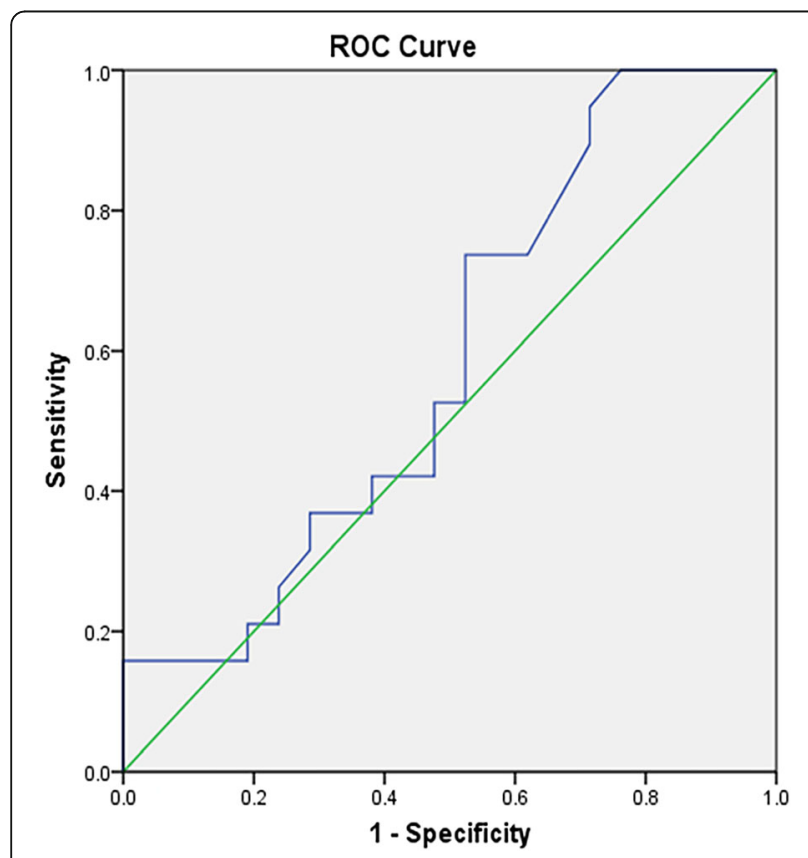

Fig. 1 ROC showing poor diagnostic performance of urinary LRG
Salo et al. [7] found urine LRG concentration when adjusted for dehydration has a better diagnostic performance especially when used in conjunction with PAS and this correlates well with the severity of appendicitis. The combination demonstrated a sensitivity of $95 \%$, specificity of $90 \%$, and AUC of 0.94 which is impressive. They also found that unadjusted LRG had equal diagnostic values to routine blood tests and any patients having high LRG but not appendicitis was improved with antibiotics alone; hence, it does not help in decisionmaking [7]. Yap et al. [9] also admit that the diagnostic ability of urine LRG by itself was mildly superior to leucocyte and neutrophil counts as it is a nonspecific acute-phase protein which is secreted in response to any inflammation. They developed a clinical prediction scoring system, namely $\mathrm{AuB}$ score, combining urine LRG (biomarker) and the component of PAS, i.e., constant pain, RIF tenderness, and pain on percussion/crying/

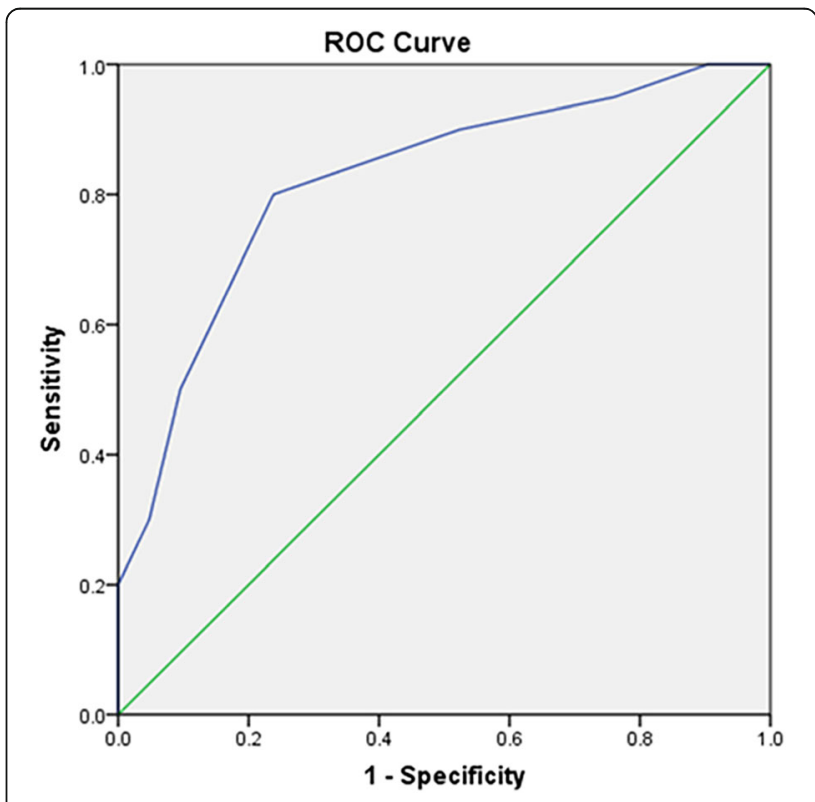

Fig. 2 ROC showing better diagnostic performance of PAS 
coughing. Sensitivity for the AuB was $97.6 \%$, specificity $37.7 \%$, negative predictive value $97.6 \%$, positive predictive value $38.3 \%$, and negative likelihood ratio 0.06 [9].

Non-operative treatment for acute uncomplicated appendicitis in children has become a standard modality. There are few metaanalysis and randomized control trials to support this treatment modality. Vardhan et al.'s metaanalysis suggested antibiotics are both safe and effective as primary treatment for children with uncomplicated appendicitis [10]. A pilot randomized control trial (RCT) by Svensson et al. [11] showed 92\% treated with antibiotics had initial resolution of symptoms and only one (5\%) case had recurrence during initial follow up suggesting safety and feasibility of non-operative management of acute appendicitis [11]. A larger multicenter, open level, noninferiority RCT is currently continuing by the same group of authors and results are awaited [12].

In our study, the treatment modality was decided by the treating physicians and surgeons. All cases of mesenteric adenitis were managed conservatively. Out of 20 cases of acute appendicitis, only 7 underwent surgery, and out of them 5 had complicated appendicitis and only 2 had inflamed appendix. So, overall, out of 15 uncomplicated appendicitis, $87 \%$ could be managed conservatively. Urinary LRG was found to be significantly elevated in 4 cases of mesenteric adenitis which were managed conservatively. On statistical analysis, urinary LRG could not show better diagnostic performance compared to PAS and hence using this as a solo biomarker for diagnosis of pediatric appendicitis or differentiating it from mesenteric adenitis using commercially available ELISA kit are not effective. Combining LRG value with PAS score also found inferior to PAS score alone. PAS score is a better diagnostic tool with AUC of 0.821 with a sensitivity of $80 \%$, a specificity of $76 \%$, PPV of $76 \%$, NPV of $80 \%$, and diagnostic accuracy of $78 \%$. Clinical judgment using PAS is still superior to any biomarker.

The study has its strength in being a prospective study on a group of homogenous patients of abdominal pain mimicking acute appendicitis, thereby removing selection bias. It is the first pilot study evaluating the diagnostic accuracy of urinary biomarkers in the Indian population. However, as a pilot study, it has an inherent limitation of being a small study. Ours being a tertiary care hospital receives a smaller number of similar patients as most get managed at periphery level and also due to strict inclusion criteria, which is based on ultrasonography, we could not include many such patients.

\section{Conclusion}

Diagnostic accuracy of urinary LRG as a sole biomarker for acute appendicitis is questionable. There was no specific cut-off value of urinary LRG which will differentiate mesenteric adenitis and acute appendicitis. With 78\% diagnostic accuracy PAS score $(>6)$ is still a better tool for the diagnosis of acute appendicitis. Elevated urinary LRG has no role in deciding the surgical intervention as most of uncomplicated appendicitis can be managed conservatively.

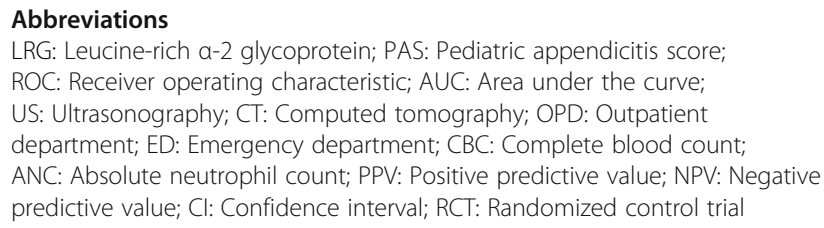

\section{Acknowledgements}

The sincere cooperation of all the faculty and residents in the department who contributed to the care of the patients is gratefully acknowledged.

\section{Authors' contributions}

Study conception and design: SKM and BBT. Data acquisition: SKM and BBT. Analysis and data interpretation: SKM, DB and ABP. Drafting of manuscript: SKM. Critical revision: MKM, DB, and ABP. All authors have read and approved the manuscript.

\section{Funding}

The research was an intramural funded project and the funding was provided by All India Institute of Medical Sciences (AlIMS), Bhubaneswar. The funding number is same as the IEC number, i.e., T/IM-F/17-18/36

Availability of data and materials

Available upon request.

\section{Declarations}

Ethics approval and consent to participate

The study was approved by the Institutional Ethics Committee (IEC) of AlIMS Bhubaneswar, IEC clearance number T/IM-F/17-18/36. All procedures performed in the study were in accordance with the ethical standards of the institution. Written informed consent was obtained from all cases included in the study. The project was also registered in the Clinical Trials Registry Government of India (CTRI/2018/01/01118), date, 8 August 2018 (http://ctri. nic.in/Clinicaltrials/login.php).

Consent for publication

Not applicable.

Competing interests

The authors declare that they have no competing interests.

\section{Author details}

${ }^{1}$ Department of Pediatric Surgery, All India Institute of Medical Sciences (AllM S) Bhubaneswar, Sijua, Patrapada, Bhubaneswar, Odisha 751020, India.

${ }^{2}$ Department of Biochemistry, All India Institute of Medical Sciences (AlIMS)

Bhubaneswar, Sijua, Patrapada, Bhubaneswar, Odisha 751020, India.

Received: 7 September 2020 Accepted: 15 March 2021

Published online: 15 June 2021

\section{References}

1. Toorenvliet B, Vellekoop A, Bakker R, Wiersma F, Mertens B, Merkus J, Breslau $P$, Hamming J. Clinical differentiation between acute appendicitis and acute mesenteric lymphadenitis in children. Eur J Pediatr Surg. 2011;21(02):120-3. https://doi.org/10.1055/s-0030-1267979.

2. Samuel M. Pediatric appendicitis score. J Pediatr Surg. 2002;37(6):877-81. https://doi.org/10.1053/jpsu.2002.32893.

3. Chadha R, Khan NA. Clinical scoring systems \& radiologic imaging in the diagnosis of pediatric appendicitis. Indian Pediatr. 2016;53(3):201-2. 
4. Brenner $D$, Elliston $C$, Hall E. Estimated risks of radiation-induced fatal cancer from pediatric CT. AJR Am J Roentgenol. 2001;176(2):289-96. https://doi. org/10.2214/ajr.176.2.1760289.

5. Kentis A, Ahmed S, Kurek K, et al. Detection and diagnostic value of urine Leucine-rich alpha-2-glycoprotein in children with suspected acute appendicitis. Ann Emerg Med. 2012;60:78-83 e1.

6. Kharbanda AB, Rai AJ, Cosme Y, Liu K, Dayan PS. Novel serum and urine markers for pediatric appendicitis. Acad Emerg Med. 2012;19(1):56-62. https://doi.org/10.1111/j.1553-2712.2011.01251.x.

7. Salo M, Roth B, Stenstrom P, Arnbjornsson E. Urinary biomarkers in pediatric appendicitis. Pediatr Surg Int. 2016;32(8):795-804. https://doi.org/10.1007/ s00383-016-3918-x.

8. Quigley AJ, Starface S. Ultrasound assessment of acute appendicitis in pediatric patients: methodology and pictorial overview of findings seen. Insights Imaging. 2013;4(6):741-51. https://doi.org/10.1007/s13244-013-02 75-3.

9. Yap TL, Fan JD, Chen Y, Ho MF, Choo CSC, Allen J, Low Y, Jacobsen AS, Nah SA. A novel noninvasive appendicitis score with a urine biomarker. J Pediatr Surg. 2019;54(1):91-6. https://doi.org/10.1016/j.jpedsurg.2018.10.025.

10. Vardhan KK, Neal KR, Lobo DN. Safety and efficacy of antibiotics compared with appendicectomy for treatment of uncomplicated acute appendicitis: meta-analysis of randomized controlled trials. BMJ. 2012;344(apr05 1):e2156. https://doi.org/10.1136/bmj.e2156.

11. Svensson JF, Patkova B, Almström M, Naji H, Hall NJ, Eaton S, Pierro A, Wester T. Nonoperative treatment with antibiotics versus surgery for acute nonperforated appendicitis in children: a pilot randomized controlled trial. Ann Surg. 2015;261(1):67-71. https://doi.org/10.1097/SLA.0000000000000835.

12. Hall NJ, Eaton $\mathrm{S}$, Abbo O, et al. Appendectomy versus non-operative treatment for acute uncomplicated appendicitis in children: study protocol for a multicentre, open-label, non-inferiority, randomized controlled trial. BMJ Paediatr Open. 2017;1 (1):bmjpo-2017-000028.

\section{Publisher's Note}

Springer Nature remains neutral with regard to jurisdictional claims in published maps and institutional affiliations.

\section{Submit your manuscript to a SpringerOpen ${ }^{\circ}$ journal and benefit from:}

- Convenient online submission

- Rigorous peer review

- Open access: articles freely available online

- High visibility within the field

- Retaining the copyright to your article

Submit your next manuscript at $\boldsymbol{\nabla}$ springeropen.com 